\title{
Properties of nonuniform grids used in ocean general circulation models
}

\author{
A. M. Treguier \\ Laboratoire de Physique des Océans, Institut Français de Recherche pour l'Exploitation de la Mer, \\ CNRS-UBO, Plouzane, France \\ J. K. Dukowicz \\ Los Alamos National Laboratory, Los Alamos, New Mexico \\ K. Bryan \\ Geophysical Fluid Dynamics Laboratory, Princeton University, Princeton, New Jersey
}

\begin{abstract}
Ocean general circulation models frequently use nonuniform grids, especially in the vertical direction. This paper clarifies the implications of using such grids on the consistency and accuracy of numerical schemes. It is emphasized that numerical schemes maintain their order of accuracy on a nonuniform grid provided the grid can be related to a smooth mapping. Additional metric terms appear in the truncation error, which should not be interpreted simply as a numerical diffusion.
\end{abstract}

\section{Introduction}

Modeling the oceanic circulation is made difficult by the broad range of important spatial scales. Both the basin scale (thousands of kilometers) and the scale of narrow straits and boundary currents (tens of kilometers) need to be represented in a numerical model. In the vertical the gradients of density and tracers are often concentrated in thin layers just below the surface mixed layer or in the thermocline. Because of computational limitations, the resolution is never sufficient and ocean modelers have been concerned by the effects of the truncation errors of the numerical schemes they use.

Yin and Fung [1991] (herein referred to as YF), for example, argued that using a nonuniform grid could increase the truncation error of the vertical advection scheme in the Geophysical Fluid Dynamics Laboratory (GFDL) general circulation model [Bryan, 1969; Cox, 1984]. Their conclusions were as follows: (1) Although the scheme is second order on a regular grid, it becomes inconsistent on an irregular grid, which means the truncation error no longer tends to zero as the grid spacing $\Delta$ is refined. (2) The advection scheme has artificial numerical diffusivity when the grid is irregular. Marti et al., [1992], pointed out that the problem does not arise in the model they use, because their numerical method is based on a smooth transformation function

Copyright 1996 by the American Geophysical Union.

Paper number 96JC01753.

0148-0227/96/96JC-01753\$09.00 which brings the irregularly spaced points (in physical space) to a regular grid (in computational space).

Here we want to stress that the statement of Marti et al. [1992] applies to all models, including the GFDL model. YF's demonstration is valid only for the very special mapping between physical and computational space that they have chosen.

YF analyze the truncation errors of the vertical advection operator on a nonuniform grid in the GFDL model and conclude that the scheme exhibits artificial numerical diffusion. It is common practice to identify second-order spatial derivative truncation error terms with diffusion, and this is apparently what YF do. Third-order spatial derivative terms in the truncation error are typically identified with dispersion. This practice arises from the theory of first-order partial differential equations and the corresponding classification of these equations as either parabolic or hyperbolic. Parabolic equations are associated with even-order spatial derivative terms and are characterized by negative real eigenvalues; this implies that second-order moments or the total "energy" are always decreasing, and therefore these equations are "dissipative." A prime example is the diffusion equation, and therefore these equations are commonly labeled "diffusive" since they tend to "smear out" prognostic quantities (positive real eigenvalues are associated with "antidiffusion"). Hyperbolic equations, on the other hand, are associated with odd-order spatial derivative terms and are characterized by imaginary eigenvalues. These equations typically represent waves whose phase speed is wavenumberdependent, and they are therefore "dispersive." This implies that second-order moments or the total energy are conserved and prognostic quantities are merely re- 
distributed by the waves. Note, however, that these equations are typically analyzed in a uniform (Cartesian) coordinate system.

Unfortunately, truncation error terms evaluated on a nonuniform grid can give rise to spatial derivative terms associated with a represention of the equation in a nonuniform coordinate system (the so-called metric terms) and not associated with the above classification. Classification by the order of the spatial derivative terms is therefore not reliable, and computation of the eigenvalues is too difficult. It is therefore important to test for the behavior of the second-order moments to conclude whether truncation errors associated with a particular scheme are diffusive or dispersive (or both). This is what we do in section 3.

\section{Consistency of the Scheme}

The order of accuracy of a numerical grid is the algebraic rate of decrease of the truncation error with the grid spacing $\Delta$. This notion is clearly defined on a regular grid only (when $\Delta$ is uniform). This issue has been discussed thoroughly by Thompson and Mastin [1985].

YF consider the example of vertical advection of temperature $\partial(w T) / \partial z$, where $w$ is the vertical velocity and $T$ the temperature, in the GFDL model. The placement of the variables $w$ and $T$ on the staggered grid is shown in Figure 1. The grid spacing $\Delta_{k}=z_{k-1 / 2}-z_{k+1 / 2}$ is positive, and the index $k$ grows from the surface downward. In the GFDL model the vertical advection is approximated by

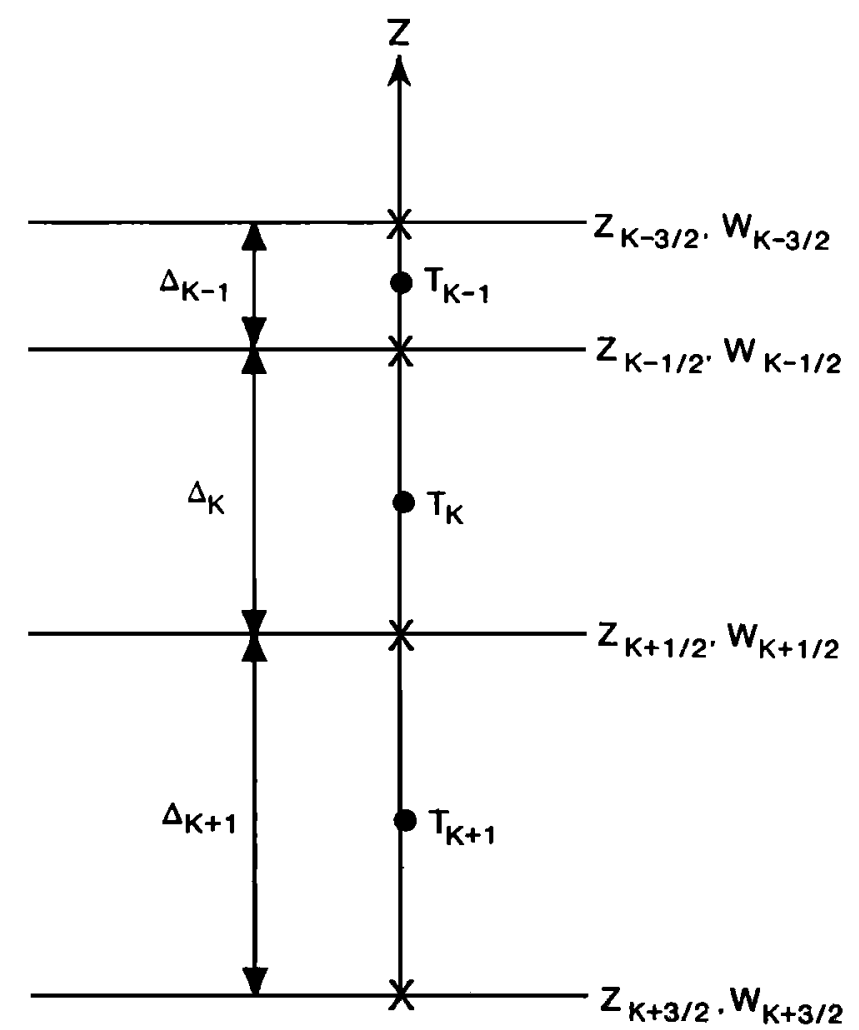

Figure 1. Position of the variables on the vertical grid.

$$
\begin{aligned}
\frac{\partial w T}{\partial z} & \approx \frac{\delta(w \bar{T})}{\delta z} \\
& =\frac{w_{k-\frac{1}{2}}\left(T_{k}+T_{k-1}\right)-w_{k+\frac{1}{2}}\left(T_{k}+T_{k+1}\right)}{2 \Delta_{k}} .
\end{aligned}
$$

YF calculate some leading order terms of the truncation error. Their equation (3) can be rewritten as

$$
\begin{gathered}
\frac{\delta(w \bar{T})}{\delta z}=\left[\frac{\partial w T}{\partial z}\right]_{k}+ \\
\left(\frac{\Delta_{k-1}+\Delta_{k+1}-2 \Delta_{k}}{4 \Delta_{k}}\right)\left[\frac{\partial T}{\partial z} w\right]_{k}+\mathcal{O}(\Delta)
\end{gathered}
$$

Because of the second term on the right-hand side, YF claim that the truncation error does not tend to zero as $\Delta$ tends to zero, therefore the scheme appears to be inconsistent (i.e., a "zeroth-order" scheme). This is not true because the truncation error in (2) contains the difference of terms involving $\Delta_{k}$. The order of the expression $\left(\Delta_{k-1}+\Delta_{k+1}-2 \Delta_{k}\right)$ is a priori unknown. A classical definition for the order of an approximation is [Ames, 1977, p.16] "if $e(x)$ is an approximation to $E(x)$ we say it is of order $n$, with respect to some quantity $\Delta_{x}$, if $n$ is the largest possible positive real number such that $|E-e|=\mathcal{O}\left(\Delta_{x}^{n}\right)$ as $\Delta_{x} \rightarrow 0$." According to this definition, (2) only shows that the order $n$ of the scheme must be $n \geq 0$. It does not demonstrate that $n$ is exactly zero.

To define the order of a scheme on a nonuniform grid one has to specify how the grid spacing $\Delta$ tends to zero, so that the order of expressions like $\left(\Delta_{k-1}+\Delta_{k+1}-2 \Delta_{k}\right)$ can be calculated. The most natural way to do this is to use a distribution function $f$ which maps physical space onto a computational space, for example the interval $[0,1]$, in which grid spacing is uniform $(\Delta=1 / N)$. The grid spacing is decreased by increasing the number of points $N$ without changing the distribution function $f$. Thompson and Mastin [1985, p.243] show that when $f$ is smooth, differentiable, and does not depend on $N$,

all difference representations maintain their order on a nonuniform grid with any distribution of points in the formal sense of the truncation error decreasing as the number of points is increased while maintaining the same relative point distribution over the field.

YF obtain a different result because they have violated this rule by choosing a very special mapping $f$ that depends on $N$ as shown below.

We take Thompson and Mastin's [1985] definition of order and assume that there exists a smooth (differentiable) function $f$ which maps the nonuniform grid (in coordinates $z$ ) to a uniform grid (in coordinates $\eta$ ):

$$
z=-H f(\eta),
$$

where $H$ is the total depth and both $f$ and $\eta$ vary between 0 and 1. Expressing $\Delta_{k}=z_{k-\frac{1}{2}}-z_{k+\frac{1}{2}}$ as a 
function of $\eta$, using the Taylor series for $f$ and the fact that the grid spacing in $\eta$ is $1 / N$, and denoting $f^{\prime}$ the derivative of $f$ with respect to $\eta$,

$$
\frac{\Delta_{k-1}+\Delta_{k+1}-2 \Delta_{k}}{4 \Delta_{k}}=\frac{1}{4 N^{2}} \frac{f^{\prime \prime \prime}}{f^{\prime}} .
$$

Here we have assumed that the mapping applies to $w$ points, $z_{k+1 / 2}=-H f\left(\eta_{k+1 / 2}\right)$, and that the $T$ points are in the middle of $w$ points as in the standard GFDL code. If the position of $T$ points is calculated taking the mapping into account, the error is smaller as follows: the factor in (4) is $1 / 8$ instead of $1 / 4$. In both cases, when $f^{\prime \prime \prime} / f^{\prime}$ is bounded as $N$ tends to infinity, the error tends to zero and the scheme is second order. The error is reduced for smooth mappings with $\left|f^{\prime \prime \prime}\right| \ll\left|f^{\prime}\right|$.

YF assume a constant stretching factor $c$. This corresponds to the mapping

$$
f(\eta)=\frac{c^{N \eta}-1}{c^{N}-1}
$$

The function $f$ depends on $N$, which violates Thompson and Mastin's [1985] criterion. The truncation error for this mapping is independent of $N$,

$$
\frac{1}{4 N^{2}} \frac{f^{\prime \prime \prime}}{f^{\prime}}=\ln ^{2}(c) / 4
$$

therefore the scheme appears to be inconsistent. However, this is merely an artifact of choosing the illbehaved mapping in (5).

\section{Numerical Diffusion}

YF note that when the grid is irregular, another term appears in the truncation error, which they interpret as a diffusion term. To discuss this issue, we consider the simplified case when $w$ is a constant. The truncation error $\epsilon$ is

$$
\epsilon=w\left[\frac{T_{k-1}-T_{k+1}}{2 \Delta_{k}}\right]-w \frac{\partial T}{\partial z} .
$$

Using classical Taylor expansion, the leading order terms are

$$
\begin{aligned}
\epsilon & \approx\left[\frac{\Delta_{k-1}+\Delta_{k+1}-2 \Delta_{k}}{4 \Delta_{k}}\right] w \frac{\partial T}{\partial z} \\
& +\left[\frac{\left(\Delta_{k-1}+\Delta_{k}\right)^{2}-\left(\Delta_{k+1}+\Delta_{k}\right)^{2}}{16 \Delta_{k}}\right] w \frac{\partial^{2} T}{\partial z^{2}} \\
& +\left[\frac{\left(\Delta_{k-1}+\Delta_{k}\right)^{3}+\left(\Delta_{k+1}+\Delta_{k}\right)^{3}}{96 \Delta_{k}}\right] w \frac{\partial^{3} T}{\partial z^{3}}
\end{aligned}
$$

The first two terms on the right-hand side resemble ones found in YF's equation (3). YF single out the second term and label it "numerical diffusion.". We do not think it is appropriate to do so.

Again expanding the grid spacings in a Taylor series using the mapping (3), we obtain

$$
\begin{aligned}
\epsilon & =\frac{f^{\prime \prime \prime}}{4 f^{\prime} N^{2}} w \frac{\partial T}{\partial z}-\frac{H f^{\prime \prime}}{2 N^{2}} w \frac{\partial^{2} T}{\partial z^{2}} \\
& +\frac{\left(H f^{\prime}\right)^{2}}{6 N^{2}} w \frac{\partial^{3} T}{\partial z^{3}}+\mathcal{O}\left(\frac{1}{N^{4}}\right)
\end{aligned}
$$

We note that all three terms are of order $N^{-2}$, and therefore all three terms on the right-hand side of (8) are formally of the same order; this leads us to suspect that these terms should not be considered independently.

It is convenient to work with a quantity related to the advection term (1) that more closely reflects the quantity actually being used in the code:

$$
\begin{array}{r}
\frac{1}{w}\left(\frac{\partial w T}{\partial z} d z\right) \approx \frac{T_{k-1}-T_{k+1}}{2} \\
\approx\left[\frac{\Delta_{k-1}+\Delta_{k+1}+2 \Delta_{k}}{4}\right] \frac{\partial T}{\partial z}+ \\
{\left[\frac{\left(\Delta_{k-1}+\Delta_{k}\right)^{2}-\left(\Delta_{k+1}+\Delta_{k}\right)^{2}}{16}\right] \frac{\partial^{2} T}{\partial z^{2}}+} \\
{\left[\frac{\left(\Delta_{k-1}+\Delta_{k}\right)^{3}+\left(\Delta_{k+1}+\Delta_{k}\right)^{3}}{96}\right] \frac{\partial^{3} T}{\partial z^{3}}}
\end{array}
$$

where $w$ was considered to be constant. Note the close resemblance between the terms in (8) and (10). Taking the coordinate transformation (3) into account, this can be rewritten as

$$
\begin{array}{r}
\frac{T_{k-1}-T_{k+1}}{2} \approx d z \frac{\partial T}{\partial z} \\
+\frac{(d z)^{3}}{6}\left[\frac{f^{\prime \prime \prime}}{H^{2} f^{\prime 3}} \frac{\partial T}{\partial z}-\frac{3 f^{\prime \prime}}{H f^{\prime 2}} \frac{\partial^{2} T}{\partial z^{2}}+\frac{\partial^{3} T}{\partial z^{3}}\right] \\
=d \eta \frac{\partial T}{\partial \eta}+\frac{(d \eta)^{3}}{6} \frac{\partial^{3} T}{\partial \eta^{3}},
\end{array}
$$

where $d z=H f^{\prime} N^{-1}$ and $d \eta=-N^{-1}$. This demonstrates that the $\partial^{2} T / \partial z^{2}$ term arises purely when transforming the $\partial^{3} T / \partial \eta^{3}$ truncation error term from $\eta$ coordinates to $z$-coordinates. In other words, the $\partial^{2} T / \partial z^{2}$ term is part of a metric term arising as a result of the coordinate transformation. This reemphasizes the point made in section 2 that discretization on a nonuniform grid is equivalent to working with a coordinate transformation and discretizing the transformed equations. Therefore the appearance of $\partial^{2} T / \partial z^{2}$ in the truncation error does not necessarily imply diffusive properties in the discretization.

To test for diffusive properties in a coordinate-independent manner, we note that the identity

$$
\begin{array}{r}
\int T(\nabla \cdot \kappa \nabla T) d V= \\
-\int \kappa|\nabla T|^{2} d V+\int \kappa T \mathbf{n} \cdot \nabla T d S
\end{array}
$$

is a coordinate-independent statement about a generic diffusion term. Thus diffusion terms must necessarily lead to negative-definite internal dissipative contributions such as the first term on the right-hand side of (12). The second term on the right-hand side is a pure 
boundary term. This is related to the conservation of second moments in ocean models. Numerical schemes used in most ocean models conserve second moments [Bryan, 1969; Blumberg and Mellor, 1987; Marti et al., 1992]. The conservation of $T^{2}$ by advection is readily demonstrated for the simplified case (10) by multiplying the operator $w \partial T / \partial z$ by $T$ and summing over the domain:

$$
\int_{-H}^{0} w T \frac{\partial T}{\partial z} d z \approx \sum_{k=1}^{N} \frac{w}{2}\left(T_{k} T_{k-1}-T_{k} T_{k+1}\right) .
$$

The terms in the discrete sum cancel except for eventual boundary contributions. A similar demonstration of second order moment conservation by the threedimensional advection operator of the GFDL model is given by, for example, Bryan [1989]. Expression (13) is the equivalent of expression (12) when applied to the advective flux. Since this contains only boundary terms, we conclude that the $\partial^{2} T / \partial z^{2}$ term in the truncation error (10) does not have the properties of a physical dissipative process.

As pointed out in the introduction, in a nonuniform coordinate system, it is not possible to associate $\partial^{2} T / \partial z^{2}$ with diffusion in a straightforward manner. Rather, one has to consider the magnitude and relative importance of all the terms in the truncation error. The relative importance of the terms in (9) clearly depends on the mapping and on the temperature profile $T(z)$. In the case of a regular grid, only the third term exists, but the total error may be larger than with the irregular grid. For example, let us consider a sharp exponential temperature profile (such as may be found in the tropics), $T(z)=T_{0} \exp (z / \lambda)$, with $\lambda=300 \mathrm{~m}$. We define a quadratic stretching, $f_{s}(\eta)=\left(1-s^{-1}\right) \eta^{2}+s^{-1} \eta$. A stretching factor of $s=1$ corresponds to a regular grid. Note that because $f^{\prime \prime \prime}=0$, the first term in (9) is zero. We have shown in Figure 2 the relative rms

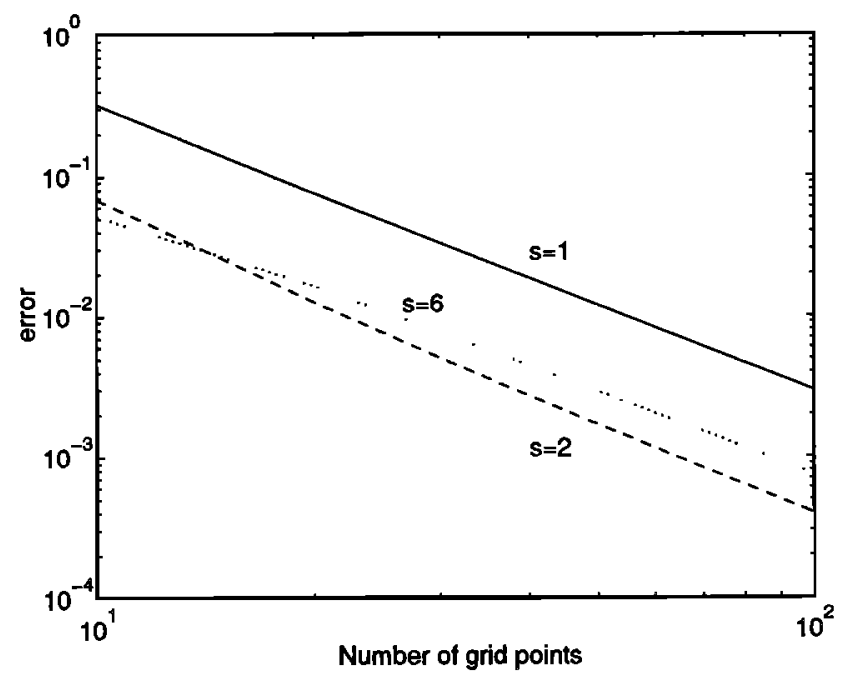

Figure 2. Relative rms error, calculated over the depth, for the numerical estimation of $\partial T / \partial z$ for three different stretched grids (see text). error as a function of the number of grid points $N$ for different stretching factors. The error ultimately always decreases like $N^{-2}$ for a sufficiently large number of points (since the scheme remains of second order on the irregular grid). As one would expect from intuition, the stretched grid has smaller error because it captures the large variations of the temperature near the surface better. In this particular case the last two terms on the right-hand side of (9) tend to cancel and reduce the error. One must not overdo the stretching, however; in our example the error again grows for stretchings larger than five. For each choice of $T(z)$ and $f(\eta)$ there is an optimal stretching factor. All these issues arise for variable grids in the horizontal, as well as the vertical, for example when using curvilinear coordinates as in the models of Blumberg and Mellor [1987] or Marti et al. [1992].

\section{Conclusions}

In the ocean, sharp gradients of properties exist in the near-surface layers and in the thermocline. Ocean modelers traditionally use irregular grids in the vertical to better resolve these features. This is a good practice. Contrary to YF's claim, this does not modify the order of the numerical scheme, nor does it render an advection scheme diffusive. It is best to define the irregular grid using a smooth stretching function as in the work by Marti et al. [1992]. Only in that case can one be sure that increasing the number of grid points in the vertical will decrease the truncation error. However, this does not necessarily give the smallest truncation error. The truncation error depends on the solution, as well as on the position of the grid points.

Global ocean models must represent regions with very different vertical profiles, and therefore the stretching of the grid is necessarily a compromise. A stretching appropriate for midlatitudes may not work as well in the tropics (e.g., a stronger stretching could decrease the truncation error even further). Conversely, even a moderate stretching may be too strong for the polar regions and could actually increase the truncation error there compared with a regular grid. Owing to the complexity of the ocean circulation, the design of vertical grids for global ocean models is an empirical process that must rely on the modeler's good judgment. This is also the reason why improvements may be achieved with adaptive meshes.

Acknowledgments. This paper was written while A. M. Treguier was enjoying an extended visit to GFDL, with support from the AOS program of Princeton University and the French CNRS. J. K. Dukowicz wishes to acknowledge the support of the DOE CHAMMP program.

\section{References}

Ames, W. F., Numerical Methods for Partial Differential Equations, Academic, San Diego, Calif., 1977. 
Blumberg, A. F., and G. L. Mellor, A description of a threedimensional coastal ocean circulation model, in ThreeDimensional Coastal Ocean Models, Coastal Estuarine Sci., vol. 4, edited by N. S. Heaps, pp. 1-16, AGU, Washington, D.C.,1987.

Bryan K., A numerical method for the study of the world ocean. J. Comput. Phys., 4, 347-376, 1969.

Bryan, K., The design of numerical models of the ocean circulation, in Oceanic Circulation Models: Combining Data and Dynamics, edited by D.L.T. Anderson and J. Willebrand, pp. 465-500, Kluwer Acad., Norwell, Mass., 1989.

Cox, M. D., A primitive equation, three-dimensional model of the ocean, GFDL Ocean Group Tech. Rep., 1, Geophys. Fluid dyn. Lab., Princeton Univ., Princeton, N.J., 1984.

Marti, O., G. Madec, and P. Delecluse, Comment on "Net diffusivity in ocean general circulation models with nonuniform grids" by F.L. Yin and I.Y. Fung, J. Geophys. Res., 97, 12,763-12,766, 1992.
Thompson, J. F., and C. W. Mastin, Order of finite difference expressions in curvilinear coordinate systems. J. of Fluids Eng., 107, 241-250, 1985.

Yin, F. L. and I. Y. Fung, : Net diffusivity in ocean general circulation models with nonuniform grids. J. Geophys. Res., 96, 10,773-10,776, 1991.

K. Bryan, Geophysical Fluid Dynamics Laboratory, NOAA, Princeton, NJ08542.

J. K. Dukowicz, Los Alamos National Laboratory, Los Alamos, NM87545.

A. M. Treguier, Laboratoire de Physique des Océans, IFREMER, BP 70, 29280 Plouzane, France. (email: treguier@ifremer.fr)

(Received June 5, 1995; revised May 22, 1996; accepted June 4, 1996.) 